\title{
Directed differentiation of human embryonic stem cells towards a pancreatic cell fate
}

\author{
J. H. Shim • S. E. Kim • D. H. Woo • S. K. Kim • \\ C. H. Oh • R. McKay • J. H. Kim
}

Received: 3 July 2006 / Accepted: 15 January 2007 / Published online: 18 April 2007

(C) Springer-Verlag 2007

\begin{abstract}
Aims/hypothesis The relative lack of successful pancreatic differentiation of human embryonic stem cells (hESCs) may suggest that directed differentiation of hESCs into definitive endoderm and subsequent commitment towards a pancreatic fate are not readily achieved. The aim of this study was to investigate whether sequential exposure of hESCs to epigenetic signals that mimic in vivo pancreatic development can efficiently generate pancreatic endodermal cells, and whether these cells can be further matured and reverse hyperglycaemia upon transplantation.

Materials and methods The hESCs were sequentially treated with serum, activin and retinoic acid (RA) during embryoid body formation. The patterns of gene expression and protein production associated with embryonic germ layers and pancreatic endoderm were analysed by RT-PCR
\end{abstract}

Electronic supplementary material The online version of this article (doi:10.1007/s00125-007-0634-z) contains supplementary material, which is available to authorised users.

J. H. Shim • S. E. Kim • D. H. Woo • S. K. Kim·J. H. Kim ( $₫)$ Division of Biotechnology,

College of Life Sciences and Biotechnology, Korea University,

West Building/Room 304, Science Campus,

1, Anam-dong 5-ga, Sungbuk-goo,

Seoul 136-713, Republic of Korea

e-mail: jhkim@korea.ac.kr

C. H. Oh

Research Institute for Medical Imaging, School of Medicine,

Korea University,

Seoul 150-050, Republic of Korea

R. McKay

Laboratory of Molecular Biology,

National Institute of Neurological Disorders and Stroke,

National Institutes of Health,

Bethesda, MD, USA and immunostaining. The developmental competence and function of hESC-derived PDX1-positive cells were evaluated after in vivo transplantation.

Results Sequential treatment with serum, activin and RA highly upregulated the expression of the genes encoding forkhead box protein A2 (FOXA2), SRY-box containing gene 17 (SOX17), pancreatic and duodenal homeobox 1 (PDX1) and homeobox HB9 (HLXB9). The population of pancreatic endodermal cells that produced PDX1 was significantly increased at the expense of ectodermal differentiation, and a subset of the PDX1-positive cells also produced FOXA2, caudal-type homeobox transcription factor 2 (CDX2), and nestin (NES). After transplantation, the PDX1-positive cells further differentiated into mature cell types producing insulin and glucagon, resulting in amelioration of hyperglycaemia and weight loss in streptozotocin-treated diabetic mice.

Conclusions/interpretation Our strategy allows the progressive differentiation of hESCs into pancreatic endoderm capable of generating mature pancreatic cell types that function in vivo. These findings may establish the basis of further investigations for the purification of transplantable islet progenitors derived from $\mathrm{hESCs}$.

Keywords Activin · Differentiation - Embryoid bodies . Endoderm · Human embryonic stem cells · Hyperglycaemia . PDX1 $\cdot$ Retinoic acid $\cdot$ Serum

$\begin{array}{ll}\text { Abbreviations } \\ \text { A } & \text { activin } \\ \text { AFP } & \alpha \text {-fetoprotein } \\ \text { CDX2 } & \text { caudal type homeobox transcription factor } 2 \\ \text { EB } & \text { embryoid body } \\ \text { FGF } & \text { fibroblast growth factor } \\ \text { FOXA2 } & \text { forkhead box protein A2 }\end{array}$




$\begin{array}{ll}\text { hESC } & \text { human embryonic stem cell } \\ \text { ITS } & \text { insulin-transferrin-selenite } \\ \text { INS } & \text { insulin } \\ \text { NES } & \text { nestin } \\ \text { PAX6 } & \text { paired box protein 6 } \\ \text { PDX1 } & \text { pancreatic and duodenal homeobox 1 } \\ \text { ProINS } & \text { proinsulin } \\ \text { RA } & \text { retinoic acid } \\ \text { S } & \text { serum } \\ \text { SF } & \text { serum-free }\end{array}$

\section{Introduction}

Human embryonic stem cells (hESCs) have been proposed to be a limitless source of specific types of adult cells to be used in therapies for degenerative diseases. To date, while many studies have reported that hESCs could be efficiently induced to differentiate into mesodermal and ectodermal lineages, such as cardiomyocytes [1], endothelial cells [2], blood cells [3] and nerve cells [4], few reports have focused on the conversion of hESCs into endodermal lineages, particularly into pancreatic cell types [5-8], which may provide a limitless stock of insulin-producing cells to treat type 1 diabetes. Moreover, despite increasing knowledge on pancreatic development, there is still debate regarding the origin of beta cells and the markers that can be used to identify their progenitors [9]. Because hESCs are a useful in vitro system for lineage tracing, the development of strategies for the differentiation of hESCs into pancreatic cell types may hold the key to these questions. The relative lack of success in terms of pancreatic differentiation of hESCs, however, indicates that directed differentiation of hESCs into definitive endoderm and subsequent commitment towards a pancreatic fate are not readily achieved.

It has been shown that pancreatic cell types can be generated from mouse and non-human primate ESCs [1018]. Based on these findings, recent studies have also suggested the potential of hESCs to differentiate into insulin-producing cells through spontaneous in vitro differentiation [5] or the use of a multi-stage protocol [6]. However, several studies have claimed that insulin staining of ESC-derived cells is likely due to insulin uptake by apoptotic cells from the culture medium [19-21], suggesting that more reliable analyses should be considered in terms of beta cell physiology and from an embryological standpoint. To date, many studies using mouse ESCs or hESCs have focused mainly on a relatively late phase of differentiation, rather than addressing the dynamics of differentiation from ESCs into pancreatic endoderm. Unlike stem cells found in the adult body, hESCs are pluripotent and capable of differentiating into various cell types. Thus, early complex events leading to sequential decisions on cell fate are an essential prerequisite for pancreatic specification. In this context, it is therefore relevant to develop an efficient strategy for obtaining an enriched cell population of pancreatic endoderm as a first step for generating functional islet cell types.

More recently, the generation of pancreatic endodermal cells from hESCs was investigated based on fundamental concepts of early pancreatic specification under spontaneous differentiation [7] and by using chemically defined medium conditions plus activin A [8]. In the present study we investigated whether efficient generation of the pancreatic endoderm can be achieved from hESCs by sequential treatment of developmentally relevant factors using an in vitro system to generate embryoid bodies (EBs).

\section{Materials and methods}

Cell culture and differentiation conditions Maintenance of hESC lines (Miz-hES4 and Miz-hES6) and generation of human EBs (hEBs) were carried out as previously described [22]. Pancreatic differentiation of Miz-hES6 cells was initiated by treating hEBs sequentially with serum, activin and all-trans retinoic acid (RA; Sigma, St Louis, MO, USA) during EB formation. The hEBs were cultured in the presence of $20 \%$ fetal bovine serum (Gibco, Grand Island, NY, USA) for the first 4 days. The serum-treated hEBs were then treated with $10-100 \mathrm{ng} / \mathrm{ml}$ activin A (R\&D systems, Minneapolis, MN, USA) under serum-free conditions for the following 6 days to determine the optimal concentration of activin A for expression of the gene encoding pancreatic and duodenal homeobox 1 (PDX1). After optimisation of the activin concentration, the effects of RA $(10 \mu \mathrm{mol} / \mathrm{l})$ on pancreatic endodermal differentiation were examined by sequential treatment of hEBs with serum $(20 \%)$ for 4 days, activin $(30 \mathrm{ng} / \mathrm{ml})$ for 4 days and RA for 2 days. The hEBs grown in suspension for 10 days were dissociated by mechanical trituration, and plated on to $35 \mathrm{~mm}$ tissue culture dishes (non-coated; Corning, Corning, NY, USA) at a density of 100-150 clusters per dish (500800 cells/cluster) in insulin-transferrin-selenite (ITS) medium [23] containing fibronectin (5 $\mu \mathrm{g} / \mathrm{ml}$; Sigma). Over 10-13 days, cells that grew out of the clusters covered large areas of the culture dishes and formed spherical clusters of various sizes. The clusters were then harvested under a dissecting microscope (Stemi 2000; Zeiss, Gottingen, Germany) using a mouth pipette connected to a pulled glass capillary tube (Sigma), and were used for immunostaining and transplantation as described below. Both cell lines showed similar patterns of differentiation, and the data obtained from Miz-hES4 cells are presented in Electronic supplementary material (ESM) Fig. 1. 
Semiquantitative RT-PCR analysis Total RNA was isolated using Trizol (Invitrogen, Grand Island, NY, USA), and cDNA was synthesised from $2 \mu \mathrm{g}$ of total RNA using the SuperScript III First-Strand Synthesis Kit (Invitrogen). PCR was subsequently carried out using AccuPower PCRPremix (Bioneer, Daejeon, Korea). The primer sequences and reaction conditions used in this study are listed in ESM Table 1. Relative band intensities were determined using an image analyser (AutoChemi Bioimaging System; UVP, CA, USA). The levels of target mRNA were normalised to the signal obtained for glyceraldehyde-3-phosphate dehydrogenase $(G A P D H)$ mRNA expression.

Immunofluorescence staining Cells were fixed in $4 \%$ paraformaldehyde in PBS. The hEBs and human adult pancreatic tissues were fixed in $4 \%$ paraformaldehyde, transferred to $20 \%$ sucrose, frozen in O.C.T compound (Tissue Tek; Sakura, Tokyo, Japan) and cut into $10 \mu \mathrm{m}-$ thick sections. Immunostaining was carried out using standard protocols, and alkaline phosphatase activity stain- ing was performed with an Alkaline Phosphatase Substrate Kit III (Vector Laboratories, Burlingame, CA, USA). The primary antibodies used in the present study are listed in ESM Table 2, and additional methodological details are described in the ESM. Human pancreatic tissues were provided by the Research Institute for Medical Imaging, School of Medicine, Korea University, in accordance with the guidelines set by the Medical Research Ethical Committee (IRB No. MD0604).

Transplantation Seven-week-old male nude BALB/c mice (Charles River, Wilmington, MA, USA) were treated with a single intraperitoneal injection of streptozotocin (Sigma; $200 \mathrm{mg} / \mathrm{kg}$ of body weight). After 6 days, animals were engrafted with $1 \times 10^{6}$ cells differentiated under different culture conditions or received a sham transplantation $(10 \mu \mathrm{l}$ of culture medium) in the left subcapsular renal space. Blood glucose levels were measured every 2-4 days between 09.00 and $11.00 \mathrm{~h}$, under non-fasting conditions, with a portable glucometer (Accu-chek Active; Roche, a

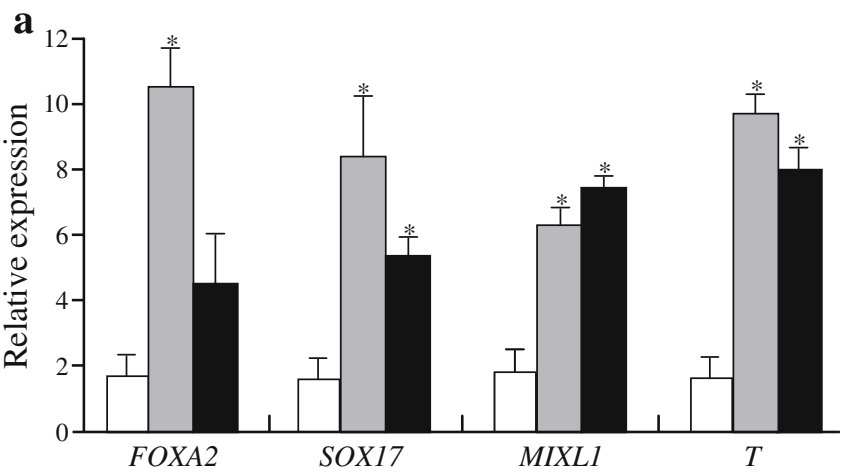

d

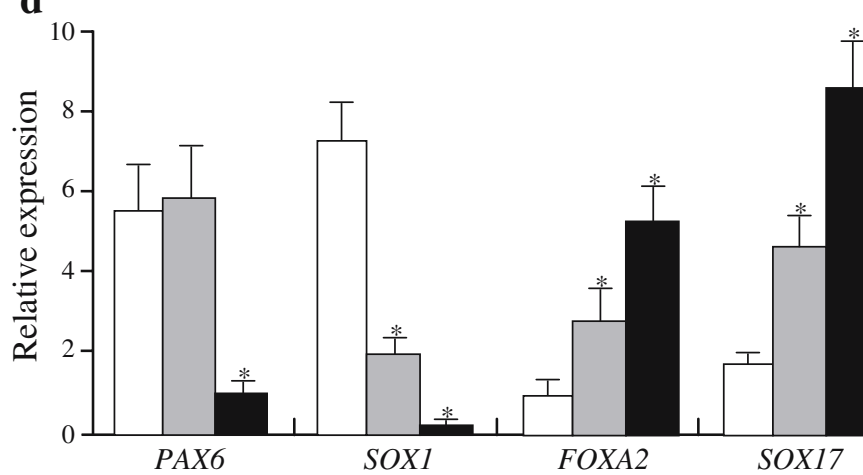

Fig. 1 Effects of serum, activin and RA on endodermal differentiation of hESCs. a The hEBs were grown in serum-free conditions for 10 days (SF10, white bars), serum-containing medium for the first 4 days followed by an additional 6 days in serum-free conditions (S4/ SF6, grey bars), or in serum for the entire 10 days (S10, black bars). hEBs differentiated under each condition were analysed for mesendodermal gene expression by RT-PCR. b Percentage and representative images of $\mathrm{T}^{+}$cells in hEBs differentiated under either SF10 (white bars) or S4/SF6 (black bars) culture conditions. DAPI 4',6-diamidino2-phenylindole dihydrochloride. c RT-PCR analysis of $P D X 1$ expres-
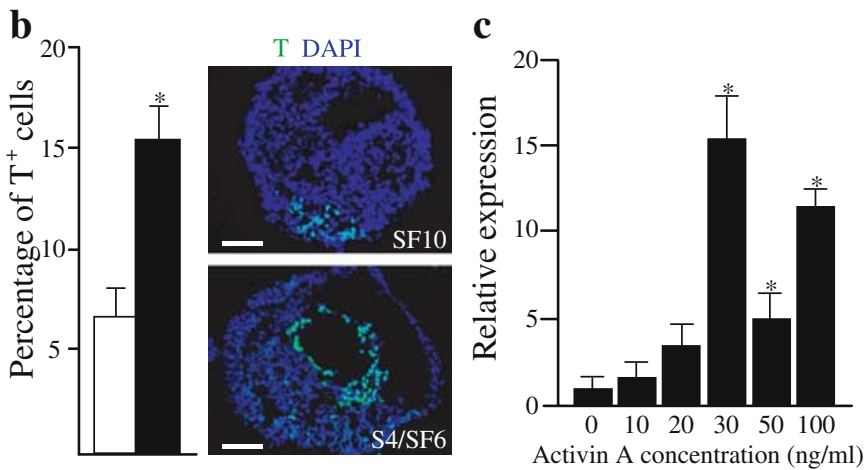
Basel, Switzerland). Body weight was monitored every 24 days. Grafts were removed 4 weeks after transplantation and were analysed by immunohistochemistry or haematoxylin/eosin staining. All experimental procedures involving animals were approved by the Korean Stem Cell Research Centre.

Statistical analysis Group means were analysed using the Student's $t$ test if only one comparison was made between two groups. Statistical differences between several groups were determined using one-way ANOVA followed by Tukey's multiple comparison tests.

\section{Results}

Differentiation of hESCs into endoderm Immunocytochemical and RT-PCR analysis revealed that the hESCs used in the present study produced OCT4, NANOG, stage-specific embryonic antigen 4 (SSEA4) and alkaline phosphatase and expressed the gene for REX1, indicating that the initial population consisted of undifferentiated ESCs (ESM Fig. 2). To investigate whether mesendoderm or endoderm could be efficiently generated from hESCs, hEBs were cultured in the absence or presence of serum and examined for the expression of mesendoderm-related genes by RTPCR analysis. As shown in Fig. 1a, when hEBs were treated with serum for 4 days and further differentiated in serum-free conditions for 6 days (S4/SF6), the expression of the genes encoding forkhead box protein A2 (FOXA2) and SRY-box containing gene 17 (SOX17) was significantly increased compared with control cultures (SF10). We also observed that the serum treatment markedly upregulated the expression of the mix 1 homeobox-like 1 gene (MIXL1), a primitive streak marker, and $T$ [human homologue of the mouse $T$ (brachyury)], which is known to be expressed in mesendoderm [24] (Fig. 1a). Although both limited (S4/ SF6) and continuous serum treatment (S10, day 0-10) strongly increased the expression of MIXL1 and $T$, we observed low levels of FOXA2 and SOX17 expression when hEBs were stimulated with serum for a prolonged period of time (S10, Fig. 1a). The effects of serum were also analysed by immunostaining using antibody against $\mathrm{T}$. $\mathrm{T}$ production was detected in few cells $(7.6 \pm 1.4 \%)$ under control conditions (SF10), and was significantly increased by limited exposure to serum $(15.4 \pm 2 \%$, S4/SF6, Fig. $1 b)$.

Differentiation of endoderm into $P D X 1^{+}$pancreatic endoderm We next attempted to examine the effects of activin A on the formation of pancreatic endoderm. Based on the finding that different concentrations of activin can induce the formation of different cell types, hEBs were stimulated for the first 4 days with serum and were subsequently further differentiated in the presence of different concentrations of activin for 6 days in serum-free conditions. Although the expression of $P D X 1$, a marker of pancreatic endoderm, was significantly increased in the presence of 30,50 and $100 \mathrm{ng} / \mathrm{ml}$ activin, the highest level of $P D X 1$ expression was observed in hEBs grown with $30 \mathrm{ng} / \mathrm{ml}$ activin (Fig. 1c). The biphasic dose-response seen could be explained by the hypothesis that the expression of activin receptor subtypes in the cells are differentially regulated in the presence of high doses of activin, as previously reported in testicular tumour cells [25].

In mouse ESCs, day 4 of EB formation is thought to correspond to the end of gastrulation in mouse embryos [17]. Based on the findings that RA signalling is necessary for pancreatic specification during this period, and that hESCs divide more slowly than mouse ESCs, we exposed $\mathrm{hEBs}$ pretreated with serum and activin $(30 \mathrm{ng} / \mathrm{ml})$ to $\mathrm{RA}$ (10 $\mu \mathrm{mol} / \mathrm{l})$ during the last 2 days (day $8-10)$ of hEB formation. Compared with control cultures (SF10) and cultures treated with serum and activin (S/A), the RA treatment (S/A/RA) significantly downregulated the expression of the neuroectodermal genes paired box gene 6 (PAX6) and $S O X 1$ (Fig. 1d). In contrast, the expression of FOXA2 and SOX17 was significantly upregulated by RA treatment (Fig. 1d). Interestingly, we observed undetectable or very low levels of MIXL1 and $T$ in the S/A/RA-treated hEBs, even though FOXA2 and SOX17 were expressed at high levels (Fig. 1d). Furthermore, together with a marked increase of $P D X 1$ expression, RA treatment upregulated the expression of the gene encoding homeobox HB9 (HLXB9) (Fig. 1d), which is expressed in the early pancreatic bud prior to $P D X 1$ expression.

It is well known that EBs consist of a cluster of three embryonic germ layers and an outer layer of primitive endoderm that produces $\alpha$-fetoprotein (AFP) and GATA4 $[26,27]$. After 10 days of EB formation, many, but not all, of the control EBs formed three-dimensional aggregates, each with a distinguishable outer layer (Fig. 2a, arrowheads). Immunostaining of frozen $\mathrm{hEB}$ sections revealed that $\mathrm{AFP}^{+} \mathrm{GATA}^{+}$cells were confined to an outer layer in the control hEBs (Fig. 2c). In contrast, S/A/RA-treated EBs had a different morphology compared with the control EBs, displaying a compact morphology with a relatively smooth surface and no prominent outer cell layer (Fig. 2b). Additionally, $\mathrm{AFP}^{+} \mathrm{GATA}^{+}$cells were barely detectable on the surface of the S/A/RA-treated EBs, while AFP ${ }^{-}$ GATA $4^{+}$cells were frequently found in the inner part of the hEBs (Fig. 2d). We next stained the EBs with antibodies against SOX17 and FOXA2. Although SOX17 ${ }^{+}$cells were found in both control and S/A/RA-treated EBs, increased numbers of SOX $17^{+}$cells were observed in S/A/RA-treated EBs, and the majority, but not all, of the $\mathrm{SOX} 17^{+}$cells were 

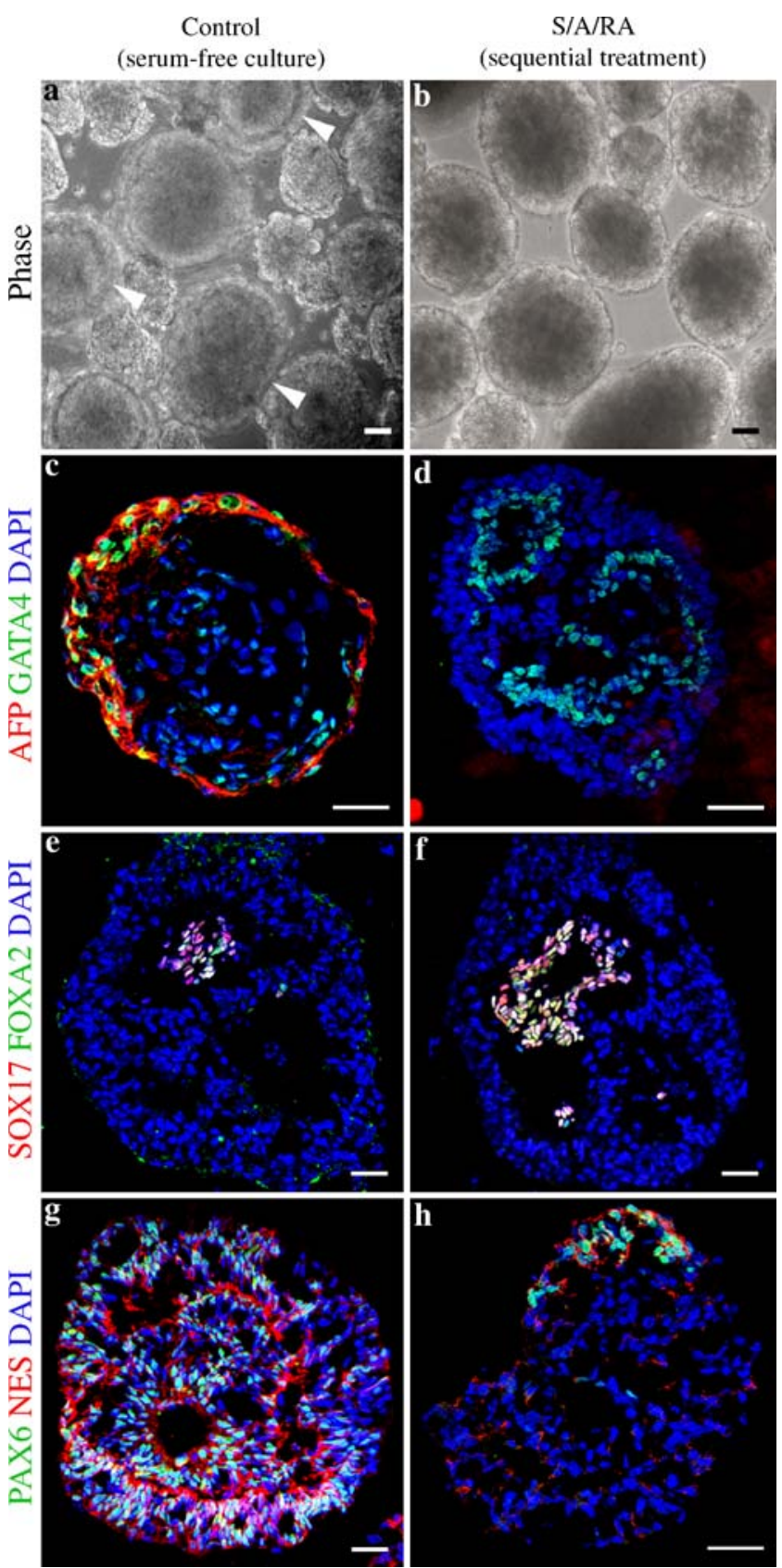

Fig. 2 Induction of endodermal differentiation during EB formation. a, b Phase-contrast morphologies of EBs differentiated under either control (serum-free) conditions (a) or by sequential treatment with $\mathrm{S}$, $\mathrm{A}$ and RA (S/A/RA) (b). Note that control EBs show distinguishable outer layers at the periphery of the aggregates (a, arrowheads). c-h Representative immunofluorescence images demonstrating the production of proteins associated with primitive and definitive endoderm (AFP/GATA4 and SOX17/FOXA2), and neuroectoderm (PAX6/NES). Note that immunoreactivity to both AFP and GATA4 is evident at the periphery of the control hEBs (c), whereas many $\mathrm{AFP}^{-} \mathrm{GATA}^{+}$cells are visible inside the S/A/RA-treated hEBs (d). Few cells producing both SOX17 and FOXA2 are seen in the control EB (e), but a large tube-like structure producing SOX17 and FOXA2 is seen in the S/A/RA-treated EB (f). S/A/RA treatment decreases formation of $\mathrm{PAX}^{+} \mathrm{NES}^{+}$neuroectoderm $(\mathbf{g}, \mathbf{h})$. DAPI 4',6-diamidino-2-phenylindole dihydrochloride. Scale bars: $50 \mu \mathrm{m}$ co-labelled with anti-FOXA2 antibody (Fig. 2e,f). Consistent with our RT-PCR results, compared with control EBs, very few S/A/RA-treated hEBs produced both PAX6 and nestin (NES; Fig. 2g,h).

Quantitative evaluation of endoderm-related protein production also revealed that, compared with serum-free culture conditions, the number of cells that were immunoreactive for FOXA2, SOX17, GATA4, PDX1 and caudal type homeobox transcription factor 2 (CDX2) was significantly increased in hEBs by treatment with S/A, and was highest in hEBs treated with S/A/RA (Fig. 3a). Consistent with RNA analyses (Fig. 1d), compared with S/A treatment, S/A/RA treatment resulted in a considerable decrease in the number of $\mathrm{T}^{+}$cells (Fig. 3a). Furthermore, immunostaining using anti-humanPDX1 antibody, tested on the human pancreatic tissue (Fig. 3b), demonstrated that hEBs treated with $\mathrm{S} / \mathrm{A} / \mathrm{RA}$ exhibited almost a fivefold increase in the proportion of $\mathrm{PDX}^{+}$cells compared with control cultures, and an increase of approximately twofold compared with S/A-treated cells (Fig. 3a,c). Interestingly, almost all of the $\mathrm{PDX}^{+}$cells produced NES, while hEBs grown in serum-free conditions predominately comprised $\mathrm{NES}^{+}$cells that did not produce PDX1, reflecting a preferential differentiation into neuroectodermal cells (Fig. 3c, upper panels). In addition, the majority of $\mathrm{PDX}^{+}$cells (all culture conditions) produced FOXA2, an upstream regulator of PDX1 (Fig. 3c, lower panels). We occasionally observed that some of the $\mathrm{PDX} 1^{+}$ cells in S/A/RA-treated hEBs were closely associated with cells producing $\mathrm{CDX} 2$, a marker of the primitive gut, which was not found under serum-free or S/A conditions (Fig. 3d).

Formation of cell clusters from $P D X 1^{+}$endoderm To further investigate the NES production in $\mathrm{PDX1}^{+}$progenitors, S/A/RA-treated hEBs were partially dissociated and further differentiated in ITS medium containing fibronectin. Over the subsequent 8 days, large areas of the dish surface were covered by cells that had migrated from the dissociated $\mathrm{hEBs}$, and $\mathrm{PDX}^{+}$cell clusters were readily detected as adherent monolayer colonies, even at 1 day after EB plating (Fig. 4a). Interestingly, at day 4 after EB plating, we observed two distinct populations that could be distinguished by their immunoreactivities. In one population, $\mathrm{PDX}^{+}$cells constituted the majority of cells in the colony, and only a subpopulation of the $\mathrm{PDX}^{+}$cells produced NES (Fig. 4b). Conversely, the other population consisted primarily of $\mathrm{NES}^{+}$cells, but some of the $\mathrm{NES}^{+}$ cells produced PDX1 (Fig. 4c). At day 8 after EB plating, most of the cells in cellular aggregates were positive for both PDX1 and NES (Fig. 4d). As the clumps grew, some of the spherical clusters containing $\mathrm{PDX}^{+}$cells became detached from the plate between 10 and 13 days after hEB plating (Fig. 4e). Immunostaining and RT-PCR analyses of the clusters revealed that some, but not all, of the $\mathrm{PDX} 1^{+}$cells 
Fig. 3 Differentiation of $\mathrm{PDX}^{+}$pancreatic endoderm from hESCs. a Quantitative immunostaining analysis demonstrating the effectiveness of different conditions (SF white bars, S/A grey bars, S/A/RA black bars) on mesendoderm and pancreatic endoderm differentiation. The quantity of cells expressing each marker is expressed as a percentage of the total cells counted following staining with 4',6-diamidino2-phenylindole dihydrochloride (DAPI). Values are expressed as means \pm SD $(n=30) .{ }^{*} p<0.05$ vs SF conditions; $* * p<0.05$ for comparison of S/A and S/A/RA conditions. b Immunostaining of a frozen section of human pancreas for PDX1. c Production of PDX1/NES (upper panels) and FOXA2/PDX1 (lower panels) in hEBs differentiated in the indicated conditions. d Co-localisation of CDX2 and PDX1 in S/A//RA-treated EBs. Scale bars: $50 \mu \mathrm{m}(\mathbf{b}, \mathbf{c})$ and $30 \mu \mathrm{m}(\mathbf{d})$

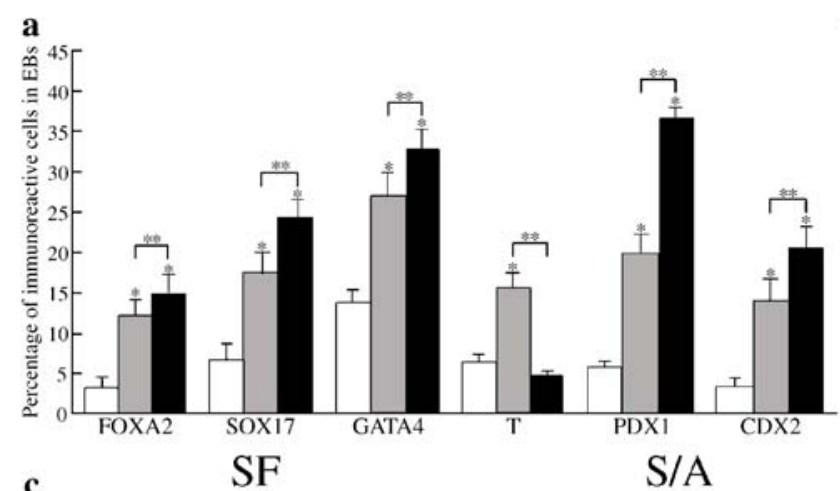

b Human pancreas
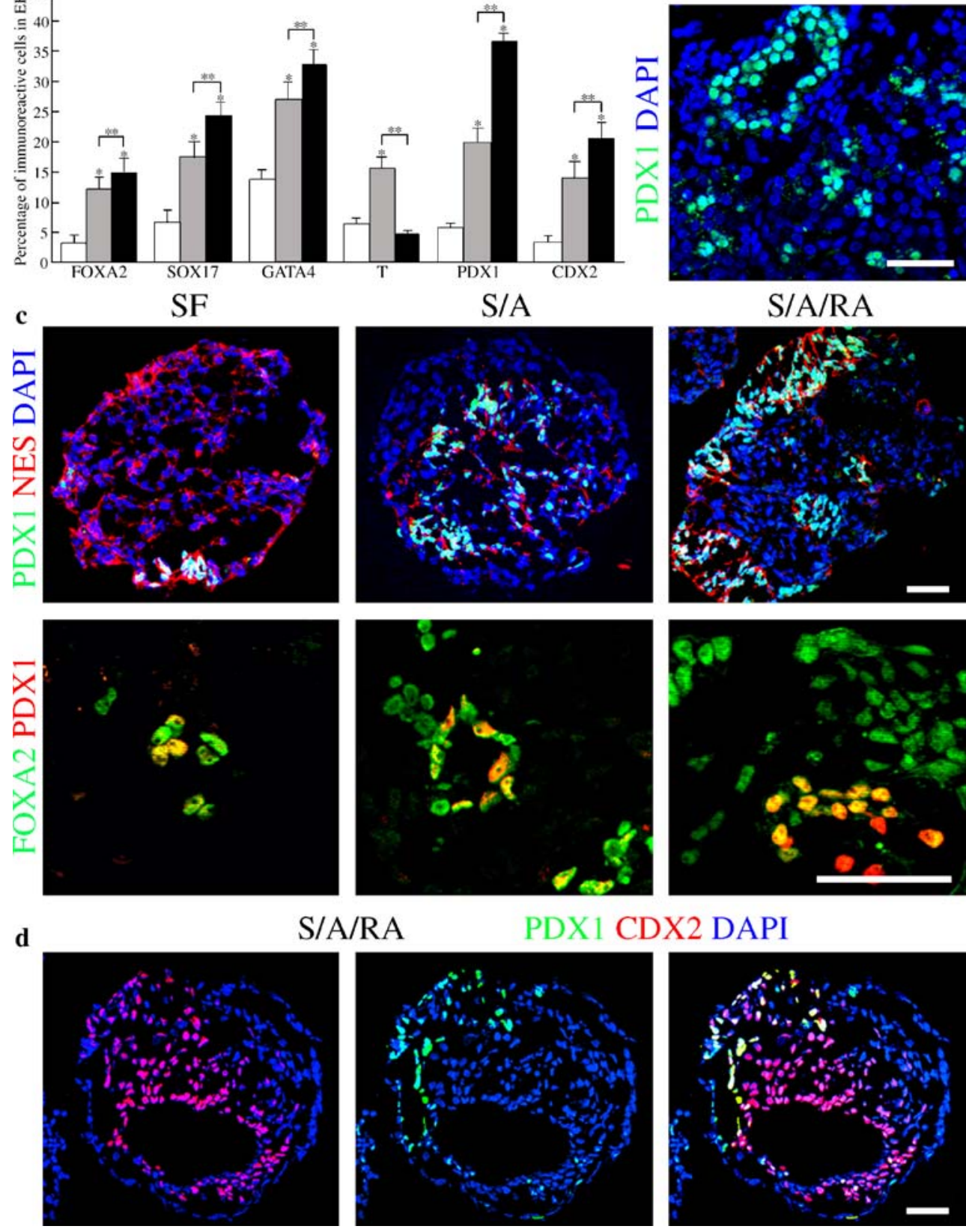

S/A/RA

PDX1 CDX2 DAPI
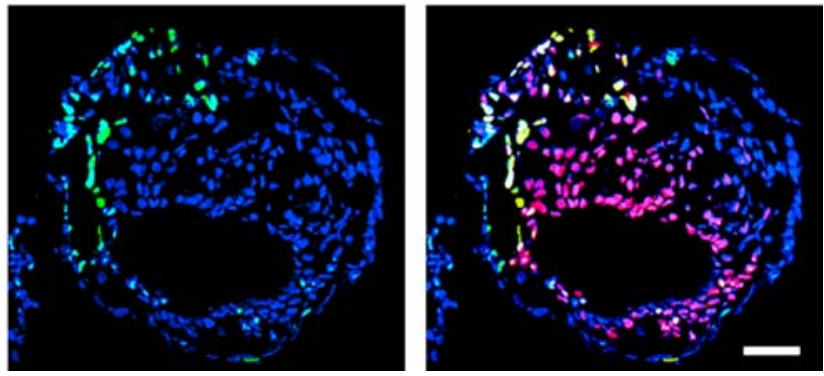

still produced NES (Fig. 4f), and a panel of genes involved in the early and late development of pancreatic islet cells were expressed at this stage (Fig. 4g). Although low levels of insulin, glucagon and somatostatin were also detected at the mRNA level, we observed no positive signals using antibodies against these proteins (data not shown).

Differentiation of $\mathrm{PDXI}^{+}$endoderm into pancreatic cell types in vivo A recent study reported that hESCs spontaneously differentiated into $\mathrm{PDX}^{+}$pancreatic progenitors, but no insulin-producing cells were detectable after the subsequent prolonged period of differentiation [7]. Similar to these findings, we were not able to detect strong insulin staining comparable to that in native islet beta cells, and observed high levels of cell death in cultures after longterm differentiation (35-40 days). Thus, to support longterm survival and differentiation, we dissociated cells or spherical clusters derived under different culture conditions (SF10, S/A and S/A/RA) and directly transplanted them underneath the kidney capsules of streptozotocin-treated mice (Fig. 5a). Diabetic mice that were sham-operated or engrafted with cells derived under SF10 conditions remained hyperglycaemic, and had glucose levels above $33.3 \mathrm{mmol} / 1$ (maximal detection limit of glucometer, 


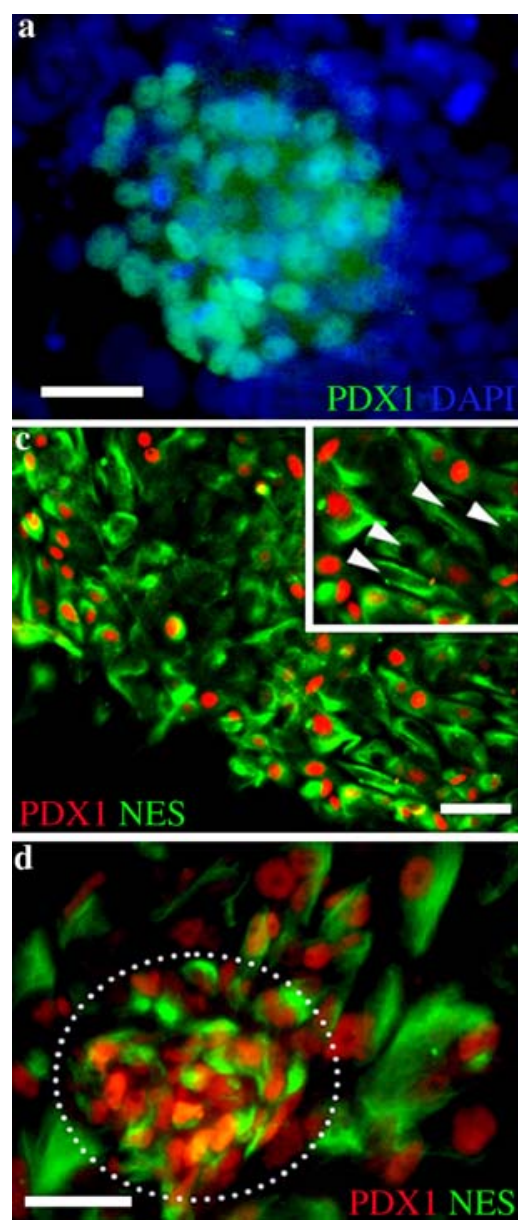

Fig. 4 Production of NES in $\mathrm{PDX} 1^{+}$pancreatic endoderm derived from S/A/RA-treated hEBs. a Immunostaining for PDX1 at day 1 of differentiation after EB plating. b-d Cells that migrated from hEBs immunostained for PDX1 and NES at day $4(\mathbf{b}, \mathbf{c})$ and day 8 (d) of differentiation after EB plating. Arrowheads in $\mathbf{b}$ (inset) indicate cells positive for PDX1 and negative for NES. Arrowheads in $\mathbf{c}$ (inset) show cells positive for NES and negative for PDX1. The dotted circle in $\mathbf{d}$ shows cell clusters that produce both PDX1 and NES after 8 days

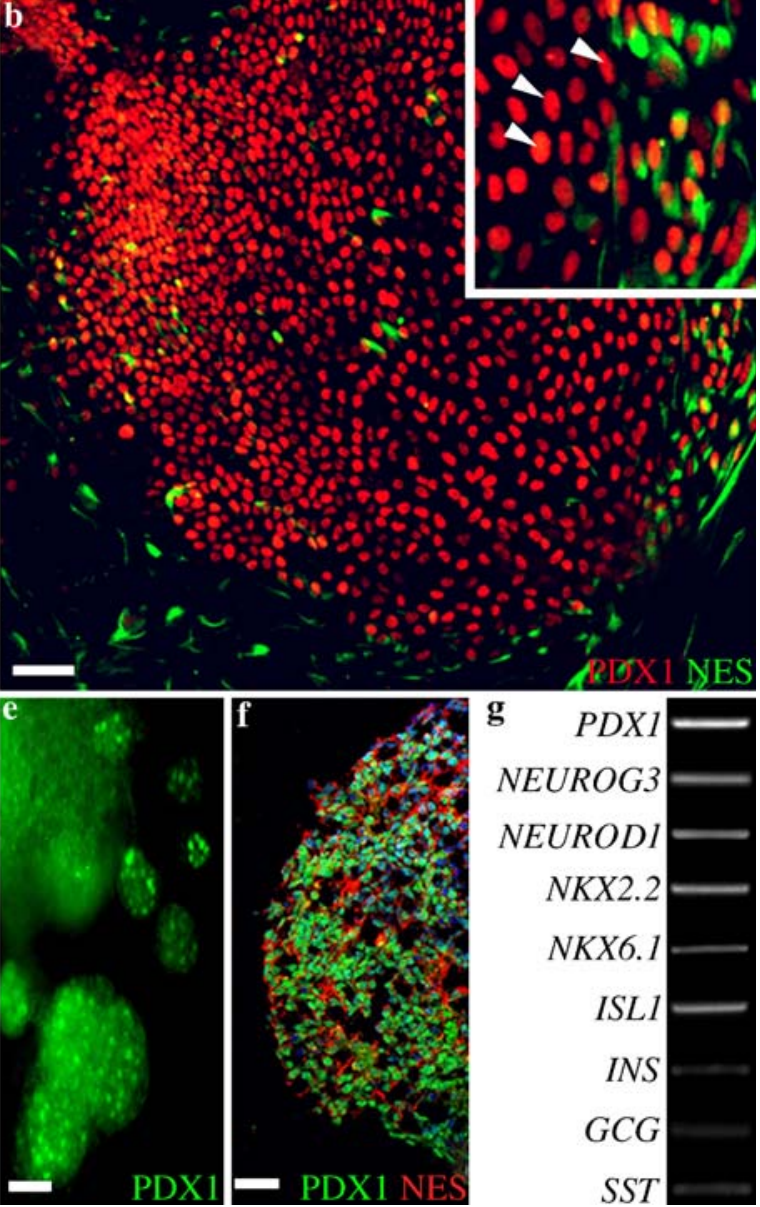

of EB plating. e Formation of spheroids producing PDX1. f Cell clusters detached from culture plates immunostained for PDX1 and NES. g RT-PCR analysis showing the expression of genes associated with early and late phases of islet differentiation in cell clusters. DAPI 4',6-diamidino-2-phenylindole dihydrochloride, $G C G$ glucagon, NEUROD1 neurogenic differentiation 1, NEUROG3 neurogenin 3, SST somatostatin, ISL1 islet 1, INS insulin. Scale bars: $25 \mu \mathrm{m}(\mathbf{a}, \mathbf{c}, \mathbf{d})$ and $50 \mu \mathrm{m}(\mathbf{b}, \mathbf{e}, \mathbf{f})$
Fig. 5b) and suffered from diabetic weight loss until they died (within 10-16 days, Fig. 5c). Conversely, mice transplanted with cells differentiated under S/A or S/A/RA conditions showed a marked reduction in blood glucose levels during the first 4 days of transplantation. However, sustained and significant decreases in glucose levels were observed only in mice engrafted with S/A/RA-derived cells, whereas blood glucose levels in the mice who received S/Aderived cells reverted to being above $33.3 \mathrm{mmol} / 116$ days after grafting (Fig. 5b). Consistent with these results, only the mice grafted with cells derived under S/A/RA conditions showed attenuated weight loss and continued to gain body weight over time (Fig. 5c).

Twenty-eight days after transplantation, we removed the kidney bearing the grafts and found that removal of grafts from the mice transplanted with $\mathrm{S} / \mathrm{A} / \mathrm{RA}$-derived cells resulted in the rapid return of hyperglycaemia, suggesting that the graft was responsible for the restoration of glucose levels (Fig. 5b). The removed grafts contained gut tubeand islet-like structures (Fig. 5d,e), suggesting endodermal differentiation in vivo. We also found numerous proinsulinpositive (ProINS ${ }^{+}$; Fig. 5f) and $\mathrm{INS}^{+}$cells (Fig. 5g) in the grafts. Additionally, the $\mathrm{INS}^{+}$cells were immunoreactive to an antibody against human nuclear antigen (Fig. 5h), indicating that these cells were derived from hESCs. INS ${ }^{+}$ cells in the grafts co-produced ProINS and C-peptide (Fig. 5i,j) and some of the $\mathrm{INS}^{+}$cells were co-stained for glucagon (Fig. $5 \mathrm{k}-\mathrm{m}$ ), suggesting that these cells synthesise insulin but are still immature, as has been described previously [28]. INS ${ }^{+} \mathrm{PDX}^{+}$cells were also often detected, suggesting the presence of more mature islet cells in the grafts (Fig. 5n). Very few $\mathrm{INS}^{+}$cells, if any, were detected 
in the grafts of mice that were sham-operated or engrafted with cells derived under SF10 or S/A conditions (data not shown). No teratomas were observed in animals transplanted with cells, although additional long-term data are needed.
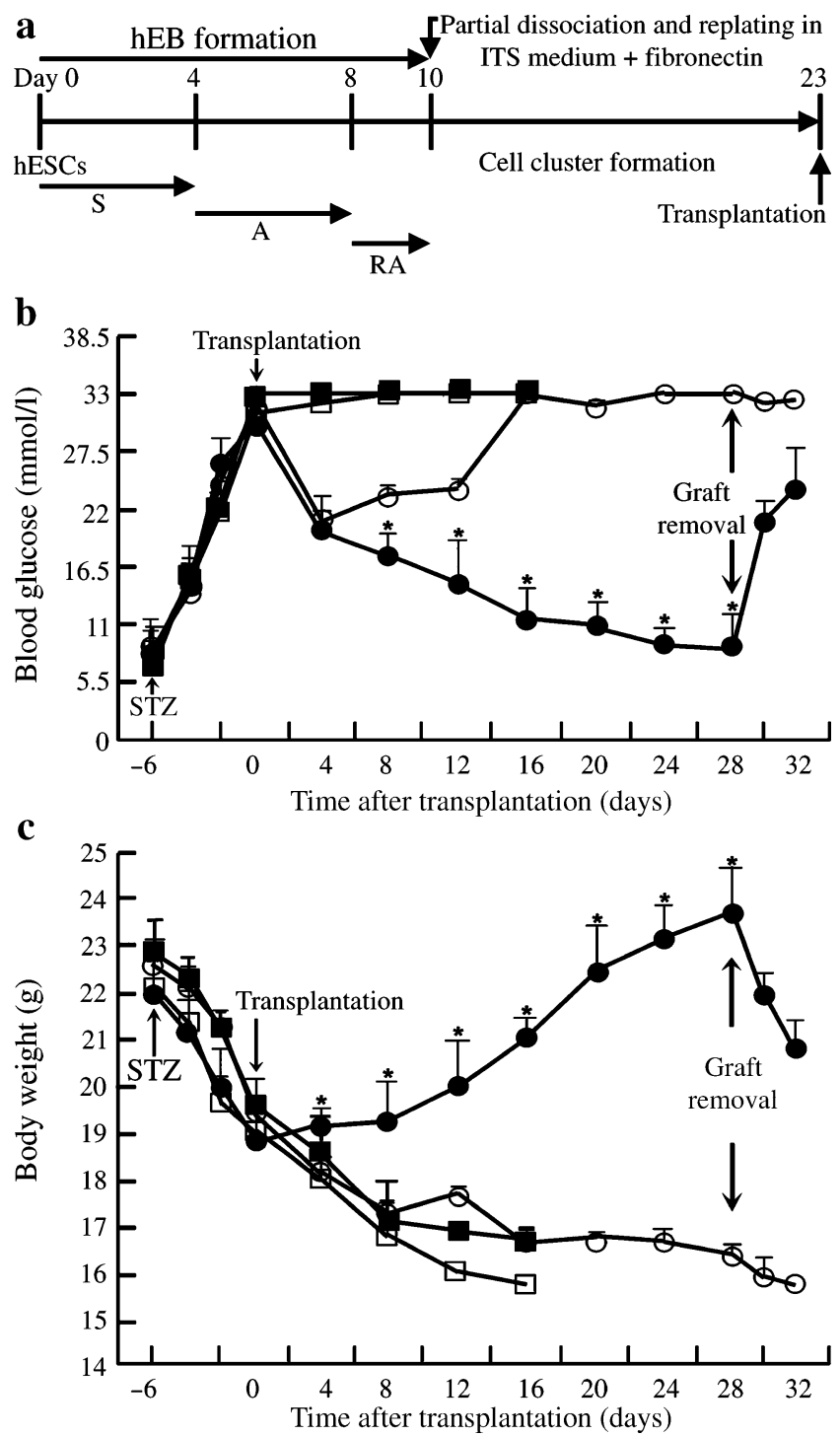

Fig. 5 Transplantation of hESC-derived $\mathrm{Pdx} 1^{+}$clusters into diabetic mice. a Schematic procedures of differentiation. $\mathbf{b}, \mathbf{c}$ Changes of blood glucose levels (b) and body weight (c) in streptozotocin-induced diabetic mice transplanted with cells derived under SF $(n=3$, closed squares), S/A ( $n=3$, open circles) or S/A/RA conditions ( $n=6$, closed circle), or in sham-operated mice ( $n=6$, open squares). d, e Haematoxylin/eosin staining showing gut tube-like (d) and islet-like structures (e) in histological sections of grafts. f Expression of proinsulin in the graft. Brown colours are positive. Cells were counterstained with haematoxylin. $\mathbf{g}-\mathbf{j}$ Immunostaining of the grafts for insulin (g), insulin/human nuclei (h), insulin/proinsulin (i) and insulin/C-peptide (j). Insets in $\mathbf{i}$ and $\mathbf{j}$ are enlarged images. Merged double-staining (red + green) appears yellow. $\mathbf{k}-\mathbf{m}$ Immunostaining for insulin (k), glucagon (I), and a merged image of insulin and glucagon immunostaining (m). $\mathbf{n}$ Immunostaining of grafts for insulin and PDX1. C-pep C-peptide, DAPI 4',6-diamidino2-phenylindole dihydrochloride, $G C G$ glucagon, $h N u c$ human nuclei, ProINS proinsulin, STZ streptozotocin. Scale bars: $50 \mu \mathrm{m}$

\section{Discussion}

Our study demonstrates that sequential exposure to serum, activin and RA stimulates the progressive differentiation of hESCs into pancreatic endoderm capable of generating mature pancreatic cell types that function in vivo. Our results show that the expression of FOXA2, SOX17, MIXL1 and $T$ is markedly increased by limited exposure to serum and activin. In mouse embryogenesis, the loss of FOXA2 or SOX17 activity results in the loss of definitive gut endoderm [29, 30], and FOXA2 is known to be a major upstream regulator of $P d x 1$ in mice $[31,32]$. In Xenopus
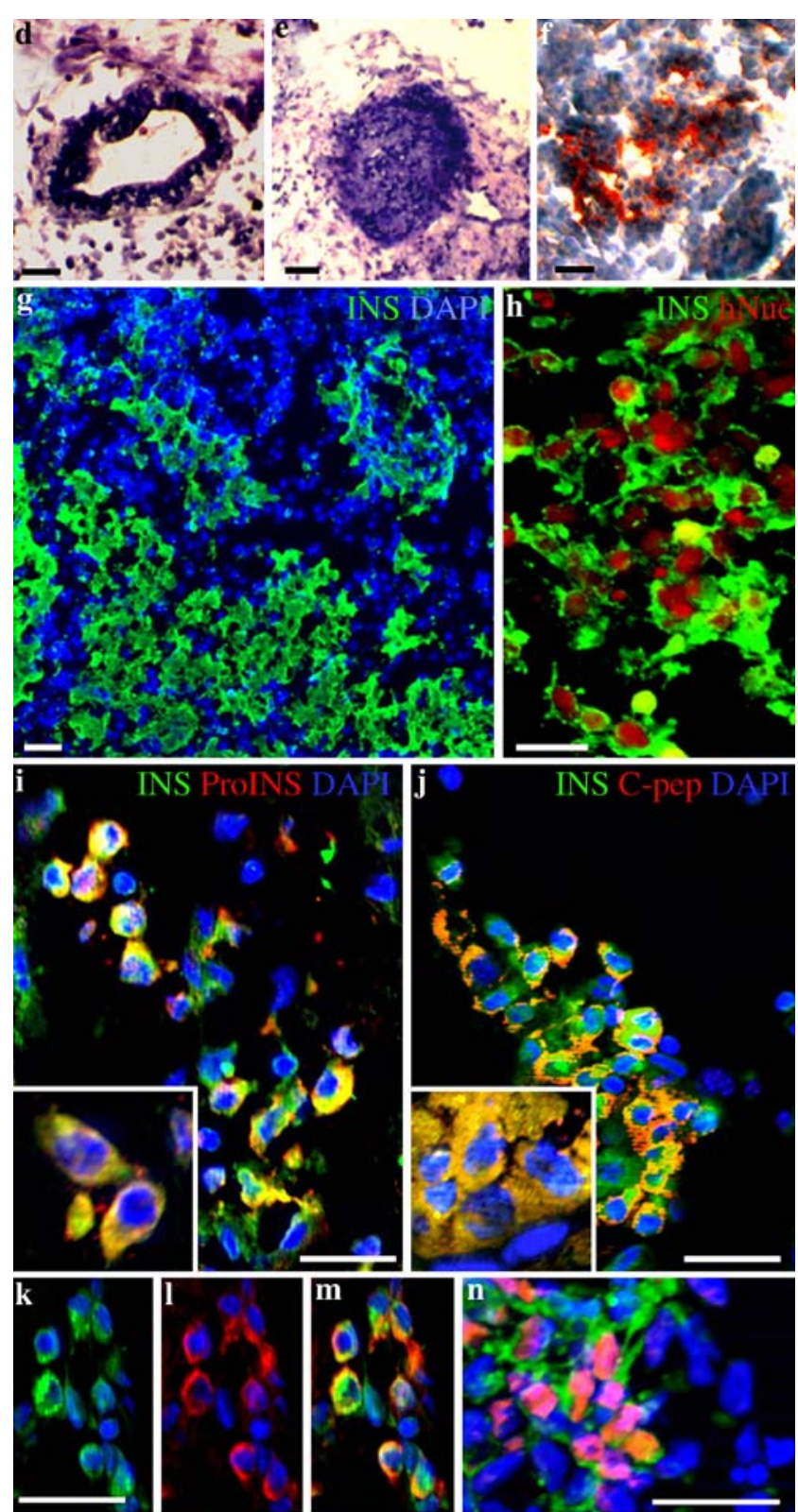

INS GCG DAPI

INS PDX1 DAPI

Fig. 5 (continued) 
laevis, Mixer, a paired-like homeobox gene, has been identified as a downstream transcriptional target of Xbra, as well as an upstream factor of Sox17b [33]. Additionally, a recent study has shown that ESCs lacking Mixl1, a murine Mix-like gene, were found to contribute poorly to gut endoderm [34]. In these embryological contexts, our data showed that differentiation of endoderm or mesendoderm can be actively directed by exposing hEBs to serum and activin. Consistent with our study, a recent study using mouse ESCs [24] reported the positive effect of serum and activin on the differentiation of definitive endoderm. A more recent study using hESCs [35] also demonstrated that, under two-dimensional differentiation conditions, a population enriched with definitive endoderm can be generated by treatment with activin $\mathrm{A}$ at a low serum concentration $(0.5 \%)$. Because we used a relatively high concentration (20\%) of serum during EB formation (three-dimensional differentiation), the optimal range of serum concentrations for endodermal differentiation seems to vary depending on the culture conditions.

RA generated from lateral plate mesoderm has recently been reported to control $P d x l$ expression in early pancreatic development in mice and zebrafish [36-38]. Our study demonstrated that RA treatment at the end of EB formation efficiently upregulated $P D X 1$ expression, together with FOXA2 and SOX17 expression, while strongly downregulating the expression of MIXL1 and $T$, both of which were previously used as markers of the mesendodermal differentiation of ESCs [35]. Although the exact mechanism underlying this phenomenon is not clear, we speculate that RA may facilitate the conversion of mesendoderm or endoderm into pancreatic endoderm in our system. This is further supported by our observation that RA increased the expression of $H L X B 9$, which is expressed in the dorsal bud of the developing mouse pancreas prior to the onset of $P d x l$ expression [39, 40]. However, we cannot rule out the possibility that RA simply increased the endodermal population at the expense of mesodermal differentiation, and the increased expression of $P D X 1$ resulted from differentiation of the enriched endodermal population.

Importantly, RA treatment significantly increased the number of $\mathrm{PDX}^{+}$cells in $\mathrm{hEBs}$, but decreased the number of $\mathrm{T}^{+}$cells. Moreover, our data also demonstrate that, at the end of hEB formation, $\mathrm{PDX}^{+}$cells co-produced FOXA2 or CDX2. Although $C d x 2$ is known to be expressed in the mid- and hindgut, and also in the placenta, a recent study has shown that $C d x 2$ and $P d x 1$ exhibit spatio-temporal overlap of expression during mouse gut development [41]. Taken together, our results suggest that RA may be a critical factor for the conversion of gut endoderm into pancreatic endoderm. Consistent with our findings, a recent report using mouse ESCs demonstrated the important role of RA in pancreatic differentiation, and also emphasised that the timing of RA treatment is crucial for the formation of $\mathrm{Pdx} 1^{+}$endoderm $[17,18]$.

Recently, several studies have demonstrated that NES is produced in human pancreatic progenitors and that insulinproducing cells can be derived from the $\mathrm{NES}^{+}$cells in vitro $[42,43]$. However, other reports have claimed that, in rodent pancreas, NES is not produced in the developing pancreatic epithelial cells [44], but is produced only in the adjacent mesenchymal cells [45] and pancreatic exocrine cell lineages [46]. Our results have shown that almost all $\mathrm{PDX}^{+}$cells present at the end of hEB formation produce NES, but these cells later become heterogeneous $\left(\mathrm{PDX}^{+}{ }^{+} \mathrm{NES}^{+}, \mathrm{PDX}^{-} \mathrm{NES}^{+}\right.$, and $\left.\mathrm{PDX}^{+}{ }^{+} \mathrm{NES}^{-}\right)$. Similarly, a recent study using clonal analysis showed that $\mathrm{NES}^{+}$ populations from the postnatal rat pancreatic islet were heterogeneous, but a small proportion of the $\mathrm{NES}^{+}$cells produced PDX1, and suggested that the $\mathrm{NES}^{+} \mathrm{PDX} 1^{+}$ population may play a role in islet formation [47]. However, it remains unclear whether the $\mathrm{PDX}^{+}$cells producing NES in our system gave rise to pancreatic endocrine cells, exocrine cells or other cell types after transplantation. Further investigations including lineagetracing studies using additional markers or genes associated with human pancreatic endocrine cells will be required to address this question.

To date, only a few studies have suggested the potential of hESCs to differentiate into insulin-producing cells [5-8]. It was previously reported that insulin-producing cells were found in EBs after spontaneous in vitro differentiation of hESCs [5]. A more recent study using the spontaneous differentiation strategy without EB formation showed that differentiated hESCs containing $\mathrm{PDX}^{+}$progenitors were able to differentiate into cells expressing islet cell markers when co-transplanted with mouse fetal dorsal pancreas [7]. Another study demonstrated that hESCs can be coaxed to differentiate into islet-like clusters by modifying the multistage method that was previously used to generate insulinsecreting clusters from mouse ESCs [48]. However, it is not clear whether the ESCs followed normal developmental sequences in these studies due to a lack of initial specification using developmentally relevant factors known to regulate pancreatic endoderm development. It has recently been shown that treatment of feeder-free hESC colonies with activin directs the differentiation of hESCs into $\mathrm{PDX}^{+}$ pancreatic endoderm, although the developmental competence of the $\mathrm{PDX}^{+}{ }^{+}$endoderm was not characterised in this study [8]. In our study, hESC-derived $\mathrm{PDX}^{+}$clusters were further differentiated into cells that produce insulin, proinsulin, C-peptide and glucagon upon transplantation. We also showed that the grafted cells ameliorated hyperglycaemia and weight loss, suggesting the competence of hESC-derived $\mathrm{PDX}^{+}$endoderm to differentiate into more mature islet cell types that function in vivo. 
Although the results presented here suggest the potential use of hESCs in the treatment of diabetes, further studies are needed both in rodent and primate systems to address the long-term safety and efficacy of these cells. Inappropriate cells may be included along with $\mathrm{PDX1}^{+}$cells upon transplantation. In particular, tumour formation is a problem associated with the grafting of differentiated ESCs. Under the conditions we used, no teratomas were seen in the graft, but long-term data are needed to show that ESC-derived cells do not form tumours.

In summary, our results show that the differentiation of pancreatic endoderm can be efficiently directed from hESCs in defined culture conditions corresponding to fundamental concepts of early pancreatic development. Our data also suggest that these cells appear to have the developmental competence to differentiate into mature pancreatic cell types that function in vivo. These findings are expected to facilitate further investigations for the purification of transplantable islet progenitors and the generation of large numbers of mature insulin-producing cells in vitro.

Acknowledgements This work was supported by a Korea Research Foundation Grant funded by the Korean government (MOEHRD) (KRF-2004-205-E00030) and a grant (SC2050) from the Stem Cell Research Centre of the 21C Frontier R\&D Programme funded by the Ministry of Science and Technology, Republic of Korea.

Conflict of interest statement The authors declare that there is no duality of interest with regard to this study.

\section{References}

1. Kehat I, Kenyagin-Karsenti D, Snir M et al (2001) Human embryonic stem cells can differentiate into myocytes with structural and functional properties of cardiomyocytes. J Clin Invest 108:407-414

2. Levenberg S, Golub JS, Amit M, Itskovitz-Eldor J, Langer R (2002) Endothelial cells derived from human embryonic stem cells. Proc Natl Acad Sci USA 99:4391-4396

3. Kaufman DS, Hanson ET, Lewis RL, Auerbach R, Thomson JA (2001) Hematopoietic colony-forming cells derived from human embryonic stem cells. Proc Natl Acad Sci USA 98:10716-10721

4. Zhang SC, Wernig M, Duncan ID, Brustle O, Thomson JA (2001) In vitro differentiation of transplantable neural precursors from human embryonic stem cells. Nat Biotechnol 19:1129-1133

5. Assady S, Maor G, Amit M, Itskovitz-Eldor J, Skorecki KL, Tzukerman M (2001) Insulin production by human embryonic stem cells. Diabetes 50:1691-1697

6. Segev H, Fishman B, Ziskind A, Shulman M, Itskovitz-Eldor J (2004) Differentiation of human embryonic stem cells into insulin-producing clusters. Stem Cells 22:265-274

7. Brolen GK, Heins N, Edsbagge J, Semb H (2005) Signals from the embryonic mouse pancreas induce differentiation of human embryonic stem cells into insulin-producing beta-cell-like cells. Diabetes 54:2867-2874

8. Yao S, Chen S, Clark J, Hao E et al (2006) Long-term selfrenewal and directed differentiation of human embryonic stem cells in chemically defined conditions. Proc Natl Acad Sci USA 103:6907-6912
9. Edlund H (2002) Pancreatic organogenesis. Developmental mechanisms and implications for therapy. Nat Rev Genet 3:524-532

10. Blyszczuk P, Czyz J, Kania G et al (2003) Expression of Pax4 in embryonic stem cells promotes differentiation of nestin-positive progenitor and insulin-producing cells. Proc Natl Acad Sci USA 100:998-1003

11. Hori Y, Rulifson IC, Tsai BC, Heit JJ, Cahoy JD, Kim SK (2002) Growth inhibitors promote differentiation of insulin-producing tissue from embryonic stem cells. Proc Natl Acad Sci USA 99:16105-16110

12. Kahan BW, Jacobson LM, Hullett DA et al (2003) Pancreatic precursors and differentiated islet cell types from murine embryonic stem cells: an in vitro model to study islet differentiation. Diabetes 52:2016-2024

13. Lester LB, Kuo HC, Andrews L, Nauert B, Wolf DP (2004) Directed differentiation of rhesus monkey ES cells into pancreatic cell phenotypes. Reprod Biol Endocrinol 2:42

14. Miyazaki S, Yamato E, Miyazaki J (2004) Regulated expression of pdx-1 promotes in vitro differentiation of insulin-producing cells from embryonic stem cells. Diabetes 53:1030-1037

15. Moritoh Y, Yamato E, Yasui Y, Miyazaki S, Miyazaki J (2003) Analysis of insulin-producing cells during in vitro differentiation from feeder-free embryonic stem cells. Diabetes 52:1163-1168

16. Soria B, Roche E, Berna G, Leon-Quinto T, Reig JA, Martin F (2000) Insulin-secreting cells derived from embryonic stem cells normalize glycemia in streptozotocin-induced diabetic mice. Diabetes 49:1-6

17. Micallef SJ, Janes ME, Knezevic K, Davis RP, Elefanty AG, Stanley EG (2005) Retinoic acid induces Pdx1-positive endoderm in differentiating mouse embryonic stem cells. Diabetes 54:301-305

18. Shi Y, Hou L, Tang F et al (2005) Inducing embryonic stem cells to differentiate into pancreatic beta cells by a novel three-step approach with activin A and all-trans retinoic acid. Stem Cells 23:656-662

19. Sipione S, Eshpeter A, Lyon JG, Korbutt GS, Bleackley RC (2004) Insulin expressing cells from differentiated embryonic stem cells are not beta cells. Diabetologia 47:499-508

20. Rajagopal J, Anderson WJ, Kume S, Martinez OI, Melton DA (2003) Insulin staining of ES cell progeny from insulin uptake. Science 299:363

21. Hansson M, Tonning A, Frandsen U et al (2004) Artifactual insulin release from differentiated embryonic stem cells. Diabetes 53:2603-2609

22. Son YS, Park JH, Kang YK et al (2005) Heat shock $70-\mathrm{kDa}$ protein 8 isoform 1 is expressed on the surface of human embryonic stem cells and downregulated upon differentiation. Stem Cells 23:1502-1513

23. Kim JH, Panchision D, Kittappa R, McKay R (2003) Generating CNS neurons from embryonic, fetal, and adult stem cells. Methods Enzymol 365:303-327

24. Kubo A, Shinozaki K, Shannon JM et al (2004) Development of definitive endoderm from embryonic stem cells in culture. Development 131:1651-1662

25. Di Simone N, Hall HA, Welt C, Schneyer AL (1998) Activin regulates betaA-subunit and activin receptor messenger ribonucleic acid and cellular proliferation in activin-responsive testicular tumor cells. Endocrinology 139:1147-1155

26. Vallier L, Reynolds D, Pedersen RA (2004) Nodal inhibits differentiation of human embryonic stem cells along the neuroectodermal default pathway. Dev Biol 275:403-421

27. Soudais C, Bielinska M, Heikinheimo M et al (1995) Targeted mutagenesis of the transcription factor GATA-4 gene in mouse embryonic stem cells disrupts visceral endoderm differentiation in vitro. Development 121:3877-3888

28. Teitelman G, Alpert S, Polak JM, Martinez A, Hanahan D (1993) Precursor cells of mouse endocrine pancreas coexpress insulin, glucagon and the neuronal proteins tyrosine hydroxylase and 
neuropeptide Y, but not pancreatic polypeptide. Development 118:1031-1039

29. Ang SL, Wierda A, Wong D et al (1993) The formation and maintenance of the definitive endoderm lineage in the mouse: involvement of HNF3/forkhead proteins. Development 119:1301-1315

30. Kanai-Azuma M, Kanai Y, Gad JM et al (2002) Depletion of definitive gut endoderm in Sox17-null mutant mice. Development 129:2367-2379

31. Wu KL, Gannon M, Peshavaria M et al (1997) Hepatocyte nuclear factor 3 beta is involved in pancreatic beta-cell-specific transcription of the pdx-1 gene. Mol Cell Biol 17:6002-6013

32. Lee CS, Sund NJ, Vatamaniuk MZ, Matschinsky FM, Stoffers DA, Kaestner KH (2002) Foxa2 controls $P d x 1$ gene expression in pancreatic beta-cells in vivo. Diabetes 51:2546-2551

33. Henry GL, Melton DA (1998) Mixer, a homeobox gene required for endoderm development. Science 281:91-96

34. Hart AH, Hartley L, Sourris K et al (2002) Mixl1 is required for axial mesendoderm morphogenesis and patterning in the murine embryo. Development 129:3597-3608

35. D’Amour KA, Agulnick AD, Eliazer S, Kelly OG, Kroon E, Baetge EE (2005) Efficient differentiation of human embryonic stem cells to definitive endoderm. Nat Biotechnol 23:1534-1541

36. Molotkov A, Molotkova N, Duester G (2005) Retinoic acid generated by Raldh2 in mesoderm is required for mouse dorsal endodermal pancreas development. Dev Dyn 232:950-957

37. Kumar M, Jordan N, Melton D, Grapin-Botton A (2003) Signals from lateral plate mesoderm instruct endoderm toward a pancreatic fate. Dev Biol 259:109-122

38. Stafford D, Prince VE (2002) Retinoic acid signaling is required for a critical early step in zebrafish pancreatic development. Curr Biol 12:1215-1220
39. Harrison KA, Thaler J, Pfaff SL, Gu H, Kehrl JH (1999) Pancreas dorsal lobe agenesis and abnormal islets of Langerhans in Hlxb9deficient mice. Nat Genet 23:71-75

40. Li H, Arber S, Jessell TM, Edlund H (1999) Selective agenesis of the dorsal pancreas in mice lacking homeobox gene Hlxb9. Nat Genet 23:67-70

41. Fang R, Olds LC, Sibley E (2006) Spatio-temporal patterns of intestine-specific transcription factor expression during postnatal mouse gut development. Gene Expr Patterns 6:426-432

42. Zulewski H, Abraham EJ, Gerlach MJ et al (2001) Multipotential nestin-positive stem cells isolated from adult pancreatic islets differentiate ex vivo into pancreatic endocrine, exocrine, and hepatic phenotypes. Diabetes 50:521-533

43. Huang H, Tang X (2003) Phenotypic determination and characterization of nestin-positive precursors derived from human fetal pancreas. Lab Invest 83:539-547

44. Duvillie B, Attali M, Aiello V, Quemeneur E, Scharfmann R (2003) Label-retaining cells in the rat pancreas: location and differentiation potential in vitro. Diabetes 52:2035-2042

45. Selander L, Edlund H (2002) Nestin is expressed in mesenchymal and not epithelial cells of the developing mouse pancreas. Mech Dev 113:189-192

46. Delacour A, Nepote V, Trumpp A, Herrera PL (2004) Nestin expression in pancreatic exocrine cell lineages. Mech Dev 121:3-14

47. Wang R, Li J, Yashpal N, Gao N (2005) Nestin expression and clonal analysis of islet-derived epithelial monolayers: insight into nestin-expressing cell heterogeneity and differentiation potential. J Endocrinol 184:329-339

48. Lumelsky N, Blondel O, Laeng P, Velasco I, Ravin R, McKay R (2001) Differentiation of embryonic stem cells to insulin-secreting structures similar to pancreatic islets. Science 292:1389-1394 